\title{
POP ZWISCHEN HISTORISMUS UND GESCHICHTSLOSIGKEIT. KANONBILDUNGEN IN DER POPULÄREN MUSIK
}

\section{Ralf von Appen, André Doehring, Helmut Rösing}

\begin{abstract}
»Popmusik gilt gemeinhin als ihrem Wesen nach ahistorisch. Jugendliche tanzen zu ihr, um sie am nächsten Tag, sobald das Nächste >Neueste eintrifft, wieder zu vergessen. [...] Show, Beat, Hingabe, Lärm, Vergnügen, Extravaganz, Gefühlsüberschwang, Furor und ,Wahnsinn<: all dies soll - ob echt oder aufgesetzt - seit jeher den wahren Kern der Popmusik ausmachen [...]. Daß dergleichen primitivistischer Vitalismus [...] aller Historisierung ebenso wie allem Historischen nicht nur gleichgültig, sondern sogar eher feindselig gegenüberstehen muß, scheint unausweichlich«(Ullmaier 1995: 7).
\end{abstract}

\section{Theoretische Annäherung}

Populäre Musik und Kanonbildung - ist das nicht ein Widerspruch in der Sache? Musik, zu der getanzt wird, die lebensnahe Funktionen erfüllt, die kaum intellektuell aufgeladen ist, die neuesten Moden folgt, die für den aktuellen Markt und den schnellen Gewinn produziert wird - kann so etwas tatsächlich kanonfähig sein?

Derartige Fragen stellen sich desto dringlicher, wenn man bedenkt, dass das aus dem Griechischen stammende Wort Kanon »Maßstab « bedeutet und seit alters her die als echt akzeptierten Textteile der Bibel bezeichnet. Echtheit steht somit als wichtiger Legitimitätsfaktor für diese Art von Kanon. Doch was ist schon echt an populärer Musik? Aus kulturkritischer Sicht so gut wie gar nichts. Aber, folgt man Theodor W. Adorno (1968), so gilt das nicht nur für die »leichte«, sondern - von wenigen Ausnahmen abgesehen - auch für die »ernste« Musik.

Das Echtheitskriterium ist also problematisch, sofern man diesen Kanonbegriff auf Musik überträgt. Verwendet man aber den Begriff Kanon nicht im 
orthodoxen, sondern im an der musikkulturellen Praxis ausgerichteten Sinn, dann ergibt sich ein durchaus anderes Bild. Musikproduktionen der Gegenwart und Vergangenheit verdichten sich im Zirkulationsprozess des allgemeinen Musiklebens zu einem kanonisierten Repertoire jener Musikstücke, die - unterschieden nach Grobstilistiken wie Klassik, Pop, Rock und Folk - als hörenswert, bedeutsam, wertvoll angesehen werden. Sie sind somit aus der Ahistorizität in den geschichtlich fundierten Kanon tradierungswürdiger kultureller »Werke« erhoben worden. Diese immer retrospektiv ausgerichtete Auswahl einzelner Stücke aus der Gesamtheit des musikkulturellen Schaffens einer Region, einer Nation, eines Kulturbereichs repräsentiert die jeweils legitime und gerne als »echt« bzw. »authentisch« bezeichnete Kultur mit starkem leitkulturellen Identitätsfaktor (vgl. Rösing 2002: 14f. u. 30).

Zur theoretischen Annäherung an die Kanonisierung populärer Musik ist es zunächst einmal wichtig zu sehen, dass Kanonisierungen von Musik gleich welchen Stils das Ergebnis eines Zusammenspiels aller kulturellen, sozialen, ökonomischen, technischen Figurationen und Ressourcen innerhalb einer bestehenden Kultur ist und dass diese Kanonisierung verschiedene wichtige Funktionen erfüllt:

Gesellschaftlich gesehen führt sie zur Definition von bleibenden Werten und zur Festschreibung einer legitimen Kultur als kulturellem Kapital mit hohem Prestige-Wert (Bourdieu 1984: 19ff.). Für die einzelne Person verhilft Kanonbildung zur ordnenden Sinngebung im Chaos der unüberschaubaren Musikneuheiten (Ehrenzweig 1982: 9ff.). Das Bedürfnis nach Vertrautheit und nach gesellschaftlichen Gemeinschaftserlebnissen (Turner 2005: 126: »Communitas «) kann ebenso zufrieden gestellt werden wie der Wunsch nach Abgrenzung gegenüber denjenigen, die sich nicht kanonkonform verhalten und z.B. die subjektive Valenz ihrer Lieblingsmusik höher bewerten als die objektivierende Valenz des Musikkanons. Aus ökonomischer und technischer Sicht schließlich verhilft kanonisierte Musik zu dauerhaften Absatzmöglichkeiten, optimaler Wiederverwertung und medialer Neuorientierung.

Kanonisierung führt generell zur Standardisierung von kulturellen Werten und Gütekriterien. Sie hat große Bedeutung für das aktuelle Musikleben, weil sie stabilisierend wirkt. Vergangenheitsgeprägte Traditionslinien ergeben eine Art von Raster, durch das die Gegenwart wahrgenommen, kategorisiert und bewertet wird. Der Alltag wird geleitet durch die Orientierung an Vorbildern, an Maßstäben, gleichgültig, ob es sich dabei um die großen Drei der klassischen Musik (Bach, Beethoven, Mozart, vgl. Dollase/Rüsenberg/ Stollenwerk 1986: 76) oder um die unangefochtene Spitzenreiter-Rolle der Beatles in der Pop-/Rockmusik handelt. 
Diese Gedanken sollen im Folgenden systematisch und detailliert ausgeführt werden. Dazu richtet sich der Blick zunächst unabhängig von populären Musiken auf allgemeine Prozesse der musikbezogenen Kanonbildung (2), um sich dann drei konkreten Kanonisierungsformen innerhalb der Pop- und Rockkultur (3), speziell dem den Diskurs beherrschenden Kanon der »besten Alben aller Zeiten« (4) zuzuwenden. Abschnitt 5 stellt dann Thesen zur gegenwärtig beobachtbaren Zunahme kanonisierender Handlungen vor.

\section{Prozesse der musikbezogenen Kanonbildung}

Kanonbildung wird greifbar als das Ergebnis eines Interessen geleiteten Diskurses zwischen Experten, Rezipienten und der Musikwirtschaft. Experten müssen Sachorientierung, musikalische Kenner- und Könnerschaft, Aspekte der Selbstprofilierung und der Publikumsakzeptanz in Einklang bringen, um sich Gehör zu verschaffen und als ernst zu nehmende Meinungs-Multiplikatoren zu wirken. Rezipienten äußern ihr Gefallen an bestimmter Musik indirekt über Konzertbesuche, Einschaltquoten und Tonträgerkauf, direkt als Kenner und Musikliebhaber im Freundes- und Bekanntenkreis oder via Leserbrief, Internetforen usw. Die Musikindustrie greift nachhaltig in den Diskurs ein durch mediale Präsenz der von innen vertriebenen Musikprodukte (Werbung, Playlists, Hit-Tabellen) und durch ihre Angebotsstrategie (Katalogpflege, Sampler-Editionen, Remakes, Preisgestaltung).

Aus all diesen Aktivitäten resultiert ein fein gesponnenes, Kanon bildendes Diskursgewebe. In ihm ergänzen bzw. bedingen sich Tendenzen der Konstruktion und Dekonstruktion. Kein Musikkanon ist somit definitiv fixiert. Zwar weist er einen durchaus Zeiten überdauernden invarianten Kern auf; insbesondere an den Randbereichen ist aber jeder Kanon veränderbar. Neue Bewertungsaspekte, neue biografische und musikbezogene Erkenntnisse können - müssen aber nicht - jederzeit zur partiellen Änderung eines bestehenden Kanons führen. Vor allem aber sind es die kreativ Schaffenden selber, die mit ihren aktuellen Stücken, Produktionen, Auftritten dazu beitragen, dass sich ein Kanon im Verlauf der Zeit ändert und mit neueren Musiktiteln aufgefüllt wird.

Wer auch immer an der Kanondefinition teilhat, übt bewusst oder unbewusst kulturelle Macht aus. Dabei können durchaus eigennützige Motive eine Rolle spielen, ebenso aber auch ernsthaft aufklärerische Bestrebungen. Dementsprechend gibt es unterschiedliche Prinzipien der Auswahl und Benennung kanontauglicher Musikstücke. Autoritär zu nennen sind die immer wieder in Fachzeitschriften oder Internetforen veröffentlichten Bestenlisten 
von Kritikern oder anderen Experten. Prinzipiell ebenfalls autoritär, dabei aber auf historische Forschung gestützt, sind die Präferenznennungen in Sach- und Fachbüchern. Hochschätzung wird hier impliziert durch die umfangreiche Dokumentation bzw. Beschreibung der vielfachen Aktivitäten bedeutender Musikerpersönlichkeiten und durch die eingehende Analyse ihrer Werke. Empirisch bzw. demokratisch ausgerichtet sind jeweils zum Jahresende oder in größeren Abständen durchgeführte Publikumsbefragungen, wie sie von vielen Zeitschriften veröffentlicht werden.

Als besonders schwierig erweist sich die Erforschung des Ineinandergreifens aller der Mechanismen, die zur kanonwürdigen »Größe« von Musikstücken beitragen. Einige der Faktoren, die den Mechanismus der Kanonbildung allein schon aus der Sicht des musikalischen Produkts in Schwung halten, seien kurz skizziert: (1) musikstrukturelle (Form, Rhythmus, Harmonie, Melodiebildung) und direkt musikbezogene Kriterien wie Sound, Tanzbarkeit, Ereignisdichte, Wiederholungshäufigkeit, (2) ästhetische, emotionale und assoziative Komponenten, die die subjektive Valenz, die Authentizität der Botschaft (Musik als nonverbales Kommunikationsmedium) und das ganz persönliche Sich-Angesprochen-Fühlen umfassen, (3) der durch Trends und Moden bestimmte Zeitgeist, der sich im musikalischen Produkt ähnlich niederschlägt wie in allen anderen kulturellen Gütern sowie (4) rezeptionspsychologisch wichtige Aspekte, die den Komplexitätsgrad ebenso beinhalten wie die verschiedenen Gesetze zur "guten Gestalt«. Diese Faktoren lassen sich in ihrer Gesamtheit kaum analytisch und empirisch in den Griff bekommen, und ihre jeweilige Gewichtung am Ergebnis scheint von Fall zu Fall unterschiedlich zu sein. Das gibt Anlass zu der Annahme, dass Kanonbildung in mancherlei Hinsicht sehr wohl zufallsbedingt sein könnte.

Funktionen, Prinzipien und Mechanismen des Kanons bzw. der Kanonbildung verweisen jedoch auf einen wichtigen übergeordneten Faktor, der in der einschlägigen Literatur bislang kaum erwähnt worden ist. Musikalische Kanonbildung setzt, wie wohl jede Art von Kanonbildung, Geschichtsbewusstsein voraus - eine Grundhaltung, die alles Neue vor dem Hintergrund des Wissens um das bereits Gewesene rezipiert. Den Motor der Kanonbildung in klassischer wie populärer Musik bildet damit ein an kulturellen Traditionen ausgerichtetes Musikverständnis. Es spricht der Musik einen über die konkrete Situation hinausweisenden Wert zu. Musik wird als etwas Geistiges, als kulturelles Gut verstanden, das erhaltenswert ist, weil es uns etwas über uns selbst, über unsere Zeit oder über vergangene Zeiten sagt. Zur Verdeutlichung dieses Sachverhalts sei ein kurzer Exkurs in die Musikgeschichte erlaubt. 
Ausgangspunkt einer eigenständigen Entwicklung der westlichen Musik ist der Drang zur schriftlichen Aufzeichnung in einem Notationssystem, das es in dieser Form in anderen Kulturen nicht gibt. Die Schwerpunktverlagerung von mündlicher Überlieferung zu schriftlicher Werkfixierung vollzog sich vom 9. bis 16. Jahrhundert in verschiedenen Schritten bis hin zur Einzelton-Notation. Sie führte zur endgültigen Befreiung aus den Fesseln althergebrachter Stereotype und deren situativ begründeten Variantenbildungen. Sie erlaubte den Übergang vom usuellen Musizieren zur rational fundierten Komposition (vgl. Kaden 1985: 334f.).

Neuheit und Originalität werden anstelle von tradierten Melodien, Rhythmen und Formen zum Synonym für musikalische Qualität. Das musikalische Produkt erhält Werkcharakter mit dem Anspruch auf Autonomie. Es wird vielfältig reproduzierbar und ist nicht mehr funktional gebunden an einen bestimmten Aufführungsanlass. Veränderte Zirkulationsbedingungen (z.B. von der Kirche, aus dem Tanzsaal in die Konzerthalle), zunehmende Arbeitsteilung (Komponist, Interpret, Dirigent), spezifische Qualitätsmerkmale (Komposition nach musiktheoretisch vorgegebenen Regeln, Interpretation nach dem Diktat der Exaktheit) prägen das Musikleben zunehmend und führen zu seiner Professionalisierung (dazu umfassend Smudits 2002). $\mathrm{Ab}$ der Mitte des 18. Jahrhunderts übernimmt folgerichtig Musikkritik die Publikums-Aufklärung in Sachen Musik und aus dieser Zeit datieren auch die ersten Musikerbiografien, der wissenschaftliche Umgang mit Musik und die Wiederentdeckung vergangener Musikwerke, etwa Mozarts Händel-Bearbeitungen (z.B. Der Messias, s. Konrad 2004: 698) oder die spektakuläre Wiederaufführung der Bachschen Matthäuspassion durch Felix MendelssohnBartholdy in Leipzig.

Dieses starke historische Interesse an der Musik früherer Zeiten führt ab dem ausgehenden 18. Jahrhundert und der »Ausbreitung des Historismus über die Musik« (Wiora 1969a) - also der »Übermacht« des Geschichtsbewusstseins in der Musikproduktion und -rezeption - zur Herausbildung eines immer wieder aufführungswürdigen Repertoires musikalischer Werke vom Spätbarock bis zur jeweiligen Gegenwart. Dieses durch Notendruck, Aufführungen und ab dem 20. Jahrhundert auch mediale Verwertung definierte Repertoire verfestigt sich zunehmend im Kanon wertvoller Werke. Sie prägen das allgemeine Musikverständnis und legen die Kriterien für gute Musik in klingenden Beispielen fest. Jene Repertoire-Vielfalt, wie sie sich z.B. in den für den aktuellen Gebrauch neu erstellten oder kopierten Musikhandschriften bis zum Anfang des 19. Jahrhunderts anhand der Handschriften-Dokumentationen des Internationalen Quellenlexikons der Musik nachweisen lässt, wird reduziert auf einen schmalen Musikkanon, der mit 
Werken der Spätromantik endet. Zudem führen all diese Prozesse zur Aufteilung der Musik in zwei Sphären: die »ernste « und die »leichte« Musik.

Der Kanon der ernsten Musik wird zum kulturellen Kapital der gehobenen Schichten in der westlichen Welt. Er stellt für sie ein legitimes Kulturgut dar, das es - ebenso wie das darin verankerte Musikverständnis - über Schule, Hochschule und Universität zu tradieren gilt. Einen allgemein akzeptierten Kanon der »leichten « Musik gab es dagegen vorerst nicht. Gassenhauer, Schlager, Hits konnten allenfalls den Status eines Evergreens erringen. Derartige Musikstücke waren somit zwar populär, aber nicht kanontauglich, sofern sie nicht Eingang in die »ernste « Musik fanden - z.B. als Zitat im Scherzo einer Sinfonie. Diese Situation hat sich jedoch im 20. Jahrhundert nachhaltig geändert.

\section{Kanonisierungsformen in der populären Musik}

Eine Übertragung der zentralen Entwicklungsschritte auf die Sphäre der populären Musik bietet sich an. Zwar hat sich die Musikproduktion von Notation und Interpretation ins Tonstudio und auf den Computerbildschirm verlagert; materielle Grundlage der Rezeption sind heute meist Tonträger bzw. Soundfiles. Das historisch ausgerichtete Interesse an Musiktiteln aus vergangener Zeit aber ist durchaus mit der Situation der »klassischen Musik« vergleichbar - seit den frühen 1970er Jahren werden Kanonisierungstendenzen vor allem im Bereich der Pop- und Rockmusik immer offensichtlicher. Differenzieren muss man dabei jedoch zwischen verschiedenen Formen der Kanonisierung, die mit jeweils verschiedenen Publika zusammenhängen: $\mathrm{Zu}$ unterscheiden sind ein Kanon der Songs, ein Kanon der Musiker und ein Kanon der als bedeutend empfundenen Alben.

Der Song-Kanon umfasst dabei das heute tatsächlich noch »lebendige « und weithin bekannte Repertoire. So sorgt z.B. das Radio abseits des tagesaktuellen Musikgeschehens dafür, dass uns Evergreens - oftmals Songs aus der Jugend der anvisierten Zielgruppen - erhalten bleiben. Ähnlich kümmern sich Top 40-Bands oder Gruppen, die sich ausschließlich dem Schaffen einzelner Bands widmen, auf Volksfesten und Tanzveranstaltungen um die Pflege eines Bestandes klassisch gewordener Songs vergangener Jahrzehnte. Zudem tragen beständig im Fernsehen angepriesene CD-Compilations wie The Golden Hits of the '60s ihren Teil dazu bei, dass Songs wie »Nights In White Satin« oder »In The Year 2525« im Gedächtnis bleiben. Die Namen ihrer Interpreten werden jedoch vergessen, denn gemeinsam ist allen 
Formen dieser Song-Kanonisierung, dass sie nicht geschichtsorientiert sind. Weder Coverbands noch Radiosender informieren ihre Zuhörer in der Regel über Entstehungsjahre und -hintergründe, meist benennen sie nicht einmal die Titel. Die Bedeutung dieses Kanons ist vor allem für den Nicht-Experten, den »Jedermann«, nicht zu unterschätzen; für Experten dagegen spielt er fast keine Rolle, weil er keinerlei Distinktion verspricht. Das Hauptkriterium dieses Kanons ist die Beliebtheit. An den Kunstbegriff ist er nicht gekoppelt.

Eine andere Form der Kanonbildung ist gerade nicht auf einzelne Songs, sondern auf deren Schöpfer ausgerichtet. In Rock-Museen steht der Personenkult ebenso im Mittelpunkt wie in den zahllosen Musiker-Biographien, die das Angebot musikbezogener Literatur auf dem Buchmarkt dominieren, oder in biografisch orientierten Filmen wie Ray, Walk The Line oder - um noch einmal in den Bereich der Klassik zu wechseln - Amadeus. Auch TVDokumentationen (ZDF Pop Gallery, MTV Rockumentary) widmen sich meist mehr den Mythen um die Musiker als ihrem kreativen Output. Zum »Helden der Musikgeschichte « kann man im öffentlichen Bewusstsein daneben durch Auszeichnungen (etwa die Aufnahme in die Rock'n'Roll Hall of Fame), Tribute-Alben oder die besonders breitenwirksame Ver-Musicalisierung werden (Buddy Holly, Queen, Abba). Dieser Kanon erreicht vor allem den Kreis musikinteressierter Laien. Die oftmals anekdotisch aufbereiteten begleitenden Informationen bieten ihnen (bei Bedarf) einen Einstieg in die intensivere Auseinandersetzung mit Musik.

An Kultur- und Bildungsdiskurse geknüpft und damit durchaus kulturelles Kapital versprechend ist die dritte Form, der Kanon der Album-Veröffentlichungen. Dies ist der Kanon der Experten bzw. derjenigen, die sich dafür halten und das Album als popmusikalisches Äquivalent zum opus der klassischen Musik ansehen. Entsprechend wird hier mit der Begrifflichkeit des Kunstdiskurses operiert, wenn es in Musikmagazinen wie dem Rolling Stone, in speziellen Alben-bezogenen Monographien wie Allan F. Moores (1997) Buch über das Sgt. Pepper's-Album, der Buchreihe 33 1/3 oder den BegleitBooklets teurer Box-Sets darum geht, »Meisterwerke der Rockgeschichte angemessen zu würdigen und zu »verstehen« (s.u.). Hier wird die Pop- und Rockmusik aus der Ahistorizität in einen Zeiten überdauernden Diskurs der Kenner und sonstigen Meinungsmacher überführt.

Auch wenn die sogenannten Experten den Laien quantitativ unterlegen sind, ist es doch der Alben-Kanon, der als die Popkultur definierend auftritt, der Impulse für die Meinungsvermittler und -multiplikatoren in Schule, Medien und Kulturpolitik gibt, weil es den »Experten« gelungen ist, die entsprechenden Kanäle und Diskurse kultureller Legitimität zu nutzen (vgl. Regev 1994). Stärker als die anderen beiden Modi der Kanonbildung ist 
dieser Diskurs historisch orientiert, ja historistisch im Sinne Wioras zu nennen. Die hier kanonisierten »Meilensteine« werden als eigenständige Werke und Teil unseres kulturellen Erbes verehrt, sie »muss « man kennen, sie bilden den Maßstab, an dem alles Spätere sich zu messen lassen hat. Stärker als bei den anderen Kanonisierungsformen wird hier zudem bewusst kanonisiert. Der Alben-Kanon ist somit am ehesten mit den Kanons anderer hochkulturell akzeptierter Künste zu vergleichen; für den Pop- und Rockhörer repräsentiert er die »Leitkultur«. Nur bei dieser Kanonform gibt es den die Hochkultur definierenden Autonomieanspruch, der sich u.a. in nachhaltig veränderten Rezeptionsbedingungen niederschlägt. Wie dort findet sich auch die Formulierung spezifischer Qualitätsmerkmale nach rationalmusikstrukturellen sowie ästhetisch-rezeptionspsychologischen Kriterien. Zugrunde liegt all dem ein im Kern identisches Musikverständnis.

\section{Die »besten Alben aller Zeiten«}

Aus den verschiedenen Formen der Alben-Kanonisierung stechen vor allem hierarchisch organisierte 100er Listen der »besten Alben aller Zeiten « heraus, wie sie fast jedes Pop-Medium irgendwann einmal zusammenstellt. Sie erheben Anspruch auf besondere Autorität, weil sie entweder von führenden Kritikern oder einer großen Anzahl Musikinteressierter zusammengestellt werden. Über Internet, Radio oder Musikmagazin erreichen sie ein breites Publikum und werden damit viel eher zum Gegenstand einer identitätsstiftenden Diskussion als einzelne Buchveröffentlichungen.

Während die Magazine Down Beat (USA) und New Musical Express (UK) schon seit 1952 jährliche Leserbefragungen zur Kür der jeweils besten Alben, Musiker, Songs etc. durchführten, finden sich die ersten AlbumListen, die sich nicht bloß auf das vergangene Jahr, sondern auf die Gesamtheit aller Veröffentlichungen beziehen, 1971 (im englischen Magazin ZigZag) und 1974 (New Musical Express) - und damit zeitlich nicht weit entfernt von den ersten Versuchen, Rock und Pop historiographisch zu erfassen (vgl. Cohn 1969, Eisen 1969, Belz 1969). Seitdem hat das Bedürfnis, musikbezogene Listen zu erstellen und zu lesen, - nicht zuletzt durch die Verbreitung des Internets - stark zugenommen (s. dazu Abschnitt 5). Abbildung 1 veranschaulicht dies am Beispiel der auf der Internetsite www.rocklist.net zu findenden Listen. Dunkle Balken zeigen die Anzahl der Listen vom Typ »beste Alben aller Zeiten«, helle Balken die Gesamtzahl der Listen aus den Fünf-Jahres-Zeiträumen. 
Best-of-Listen 1960-2004

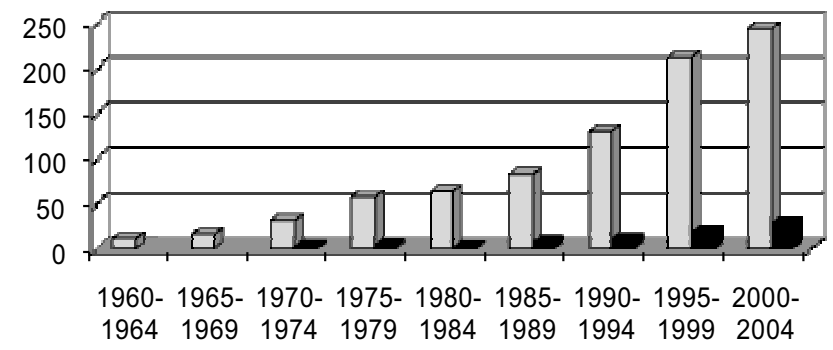

Abb. 1: Bei www.rocklist.net (14.10.2007) verzeichnete Listen, sortiert nach Datum der Veröffentlichung.

Um diesen Alben-Kanon auf seine Inhalte und seine Konstanz zu untersuchen, wurden in vorangegangenen Studien 22 solcher Listen aus den Jahren 1985-1999 bzw. sechzehn weitere Listen der Jahre 2000-2004 zusammengetragen und zu einer Meta-Liste zusammengerechnet (zum genauen Verfahren s. Appen/Doehring 2000 bzw. 2006). Die Ergebnisse sind in Abbildung 2 zusammengefasst.

Auffällig ist dabei zunächst, dass sich die Abstimmenden in hohem Maße einig sind. Von den rechnerisch möglichen 950 Plätzen wurden nur 273 besetzt. Die Übereinstimmung in der Auswahl nimmt dabei zu, je höher ein Album platziert ist: der aus der Sozialpsychologie bekannte und von Salganik/Dodds/Watts (2006), bezogen auf Musikbewertungen, eindrucksvoll nachgewiesene »The winner takes it all«-Effekt. In einem ersten Überblick lassen sich zunächst zwar banale, aber dennoch nicht zu vernachlässigende Merkmale der hier repräsentierten Musik erkennen, die als Eingangsvoraussetzung gewertet werden können: Die Alben der Top 30 enthalten Songs, die fast durchweg im 4/4-Takt stehen, selten die Länge von vier Minuten überschreiten, von den Bandmitgliedern selbst komponiert, mit englischen Texten gesungen, von »klassischer « Rockbesetzung (voc, p, g, b, dr) gespielt und bei Major-Labels nach 1964 veröffentlicht wurden. Besonders auffällig ist, dass die Musiker fast ausschließlich weiße Männer aus den USA (43\%) oder Großbritannien (52\%) sind.

Daneben springt ins Auge, dass die Jahre zwischen 1965 und 1969 offenbar als »goldenes Zeitalter gelten. Auf diesen kurzen Zeitraum entfallen $34 \%$ aller vergebenen Punkte, während die Jahre 1980-1984 für die Wählenden sehr trostlos gewesen sein müssen. Die Veröffentlichungen aus diesen Jahren erreichen zusammen nur 3,7 \% der Gesamtpunktzahl (s. Abb. 3). 


\begin{tabular}{|c|c|c|c|c|c|c|}
\hline & 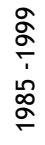 & 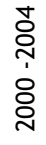 & Album & Musiker & $\Sigma$ & Jahr \\
\hline 1 & 2 & 1 & Revolver & The Beatles & 566 & 1966 \\
\hline 2 & 1 & 3 & Sgt. Pepper's Lonely Hearts... & The Beatles & 541 & 1967 \\
\hline 3 & 8 & 2 & Nevermind & Nirvana & 469 & 1991 \\
\hline 4 & 5 & 4 & The Beatles & The Beatles & 435 & 1968 \\
\hline 5 & 4 & 6 & Pet Sounds & The Beach Boys & 409 & 1966 \\
\hline 6 & 14 & 7 & Abbey Road & The Beatles & 342 & 1969 \\
\hline 7 & 12 & 8 & Dark Side Of The Moon & Pink Floyd & 336 & 1973 \\
\hline 8 & 6 & 12 & The Velvet Underground \& Nico & The Velvet Underground & 327 & 1967 \\
\hline 9 & 7 & 18 & Blonde On Blonde & Bob Dylan & 295 & 1966 \\
\hline 10 & 25 & 5 & OK Computer & Radiohead & 290 & 1997 \\
\hline 11 & 3 & 31 & Astral Weeks & Van Morrison & 268 & 1968 \\
\hline 12 & 11 & 13 & Exile On Main St. & Rolling Stones & 263 & 1972 \\
\hline 13 & 9 & 24 & What's Going On & Marvin Gaye & 249 & 1971 \\
\hline 14 & 13 & 19 & Never Mind The Bollocks... & The Sex Pistols & 242 & 1977 \\
\hline 15 & 10 & 23 & Highway 61 Revisited & Bob Dylan & 241 & 1965 \\
\hline 16 & 23 & 9 & The Joshua Tree & U2 & 236 & 1987 \\
\hline 17 & 17 & 10 & The Bends & Radiohead & 222 & 1995 \\
\hline 18 & 16 & 17 & The Stone Roses & The Stone Roses & 201 & 1989 \\
\hline 19 & 29 & 11 & London Calling & The Clash & 185 & 1979 \\
\hline 20 & 21 & 20 & Blood On The Tracks & Bob Dylan & 163 & 1975 \\
\hline 21 & 15 & 34 & Are You Experienced? & Jimi Hendrix Experience & 160 & 1967 \\
\hline 22 & 24 & 21 & The Queen Is Dead & The Smiths & 158 & 1986 \\
\hline 23 & 19 & 35 & Automatic For The People & R.E.M. & 154 & 1992 \\
\hline 24 & 38 & 14 & Rumours & Fleetwood Mac & 131 & 1977 \\
\hline 25 & 33 & 22 & Achtung Baby & U2 & 129 & 1991 \\
\hline 26 & 46 & 16 & Ten & Pearl Jam & 121 & 1992 \\
\hline 27 & 27 & 36 & Born To Run & Bruce Springsteen & 120 & 1975 \\
\hline 28 & 51 & 15 & Rubber Soul & The Beatles & 118 & 1965 \\
\hline 29 & 18 & 80 & Let It Bleed & Rolling Stones & 116 & 1969 \\
\hline 30 & 22 & 61 & (What's The Story) Morning Glory? & Oasis & 110 & 1995 \\
\hline
\end{tabular}

Abb. 2: Meta-Liste, zusammengestellt aus 38 Listen vom Typ $\gg 100$ beste Alben aller Zeiten«. Spalte eins informiert über die Position, die sich ergibt, wenn man alle Listen von 1985 bis 2004 addiert, Spalte zwei gibt den Stand von 1999 wieder, Spalte drei berücksichtigt nur Listen der Jahre 2000 bis 2004. 
Verteilung über 5-Jahres-Räume $\square$ Top 25

- Top 200

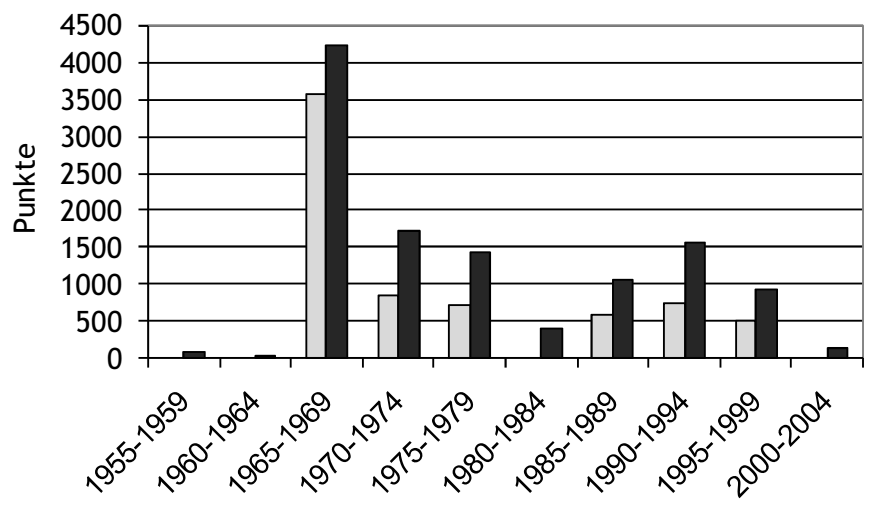

Abb. 3: Punktsummen der Alben der Meta-Liste auf Fünf-Jahres-Zeiträume

Weiterhin äußerst auffällig ist die starke Dominanz der Beatles, von denen allein vier Alben in den Top 10 vertreten sind. Insgesamt konnten sie für sieben platzierte Alben ca. ein Sechstel aller vergebenen Punkte sammeln. Im Vergleich schneiden andere immer wieder als Heroen der Popgeschichte bezeichnete Musiker wie Bob Dylan (4 Alben, 705 Punkte) oder die Rolling Stones (6 Alben, 527 Punkte) deutlich schwächer ab. Bemerkenswert sind die Ergebnisse von Nirvana (3 Alben, 516 Punkte) und Radiohead (2 Alben, 512 Punkte), deren Alben sich innerhalb weniger Jahre bereits als Klassiker etablieren konnten und die noch höher geschätzt werden als das Werk von Pink Floyd (473 Punkte) und U2 (415 Punkte). Mit nur jeweils einem einzigen in der Gesamtliste vertretenen Album schafften die Beach Boys (409 Punkte) und Velvet Underground (327 Punkte) den Sprung auf Platz 8 und 9 der beliebtesten Alben-Künstler. Die Beatles konnten ihren Anteil an allen vergebenen Punkten seit 2000 sogar von 16,6 auf 19 Prozent erhöhen. Ihr Status als Klassiker hat sich also nicht nur erhalten, sondern noch verstärkt, während Dylan, die Rolling Stones, Jimi Hendrix, Marvin Gaye oder Van Morrison signifikant an Anerkennung verloren haben (Astral Weeks sank gar um 28 Ränge).

Abgesehen davon ist eine recht hohe Kanonstabilität festzustellen: Dreizehn Alben werden sowohl in den Top 20 der Liste von 1987-1999 als auch in der Liste 1999-2004 genannt. Fünf Alben der aktuellen Top 10 waren schon in der New Musical Express-Liste von 1974 in den Top 10 vertreten (Revolver, Sgt. Pepper's, Pet Sounds, Abbey Road, Blonde on Blonde). 
Weitere wichtige Hinweise ergeben sich aus dem Vergleich dieses Listen-Kanons mit den in den USA erhobenen Verkaufszahlen (s. Abb. 4; die weltweiten Verkaufszahlen sind weniger zuverlässig, daher werden sie im Folgenden nicht näher berücksichtigt).

\begin{tabular}{|c|c|c|c|c|c|c|}
\hline & Album & Musiker & $\frac{\frac{1}{\pi}}{\stackrel{D}{2}}$ & 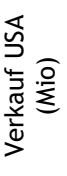 & 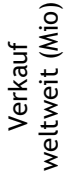 & 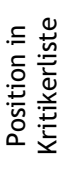 \\
\hline 1 & $\begin{array}{l}\text { Their Greatest Hits } \\
1971-1975\end{array}$ & The Eagles & 1976 & 28 & 41 & - \\
\hline 2 & Thriller & Michael Jackson & 1983 & 26 & 54 & 40 \\
\hline 3 & The Wall & Pink Floyd & 1979 & 23 & 20 & 32 \\
\hline 4 & Led Zeppelin IV & Led Zeppelin & 1971 & 22 & 30 & 39 \\
\hline 5 & Greatest Hits Vol. $1 \& 2$ & Billy Joel & 1985 & 21 & 15 & - \\
\hline \multirow[t]{4}{*}{6} & Rumours & Fleetwood Mac & 1977 & 19 & 30 & 24 \\
\hline & Back In Black & $\mathrm{AC} / \mathrm{DC}$ & 1980 & 19 & 42 & - \\
\hline & The Beatles & The Beatles & 1968 & 19 & 18 & 4 \\
\hline & Come On Over & Shania Twain & 1997 & 19 & 35 & - \\
\hline \multirow[t]{2}{*}{10} & Boston & Boston & 1976 & 17 & 18 & - \\
\hline & Bodyguard O.S.T. & Various Artists & 1992 & 17 & 37 & - \\
\hline \multirow[t]{6}{*}{12} & Cracked Rear View & Hootie \& the Blowfish & 1994 & 16 & 17 & - \\
\hline & Greatest Hits & Elton John & 1974 & 16 & 20 & - \\
\hline & Jagged Little Pill & Alanis Morisette & 1995 & 16 & 28 & 55 \\
\hline & The Beatles 1967-1970 & The Beatles & 1973 & 16 & 20 & - \\
\hline & No Fences & Garth Brooks & 2000 & 16 & 16 & - \\
\hline & Hotel California & The Eagles & 1976 & 16 & 26 & 82 \\
\hline
\end{tabular}

Abb. 4: Die meistverkauften Alben in den USA sowie weltweit (www.riaa.com/gp/ bestsellers/topalbums.asp; 21.5.2005 bzw. http://top40charts.com/chart.php?cid=25\& compag=38; 27.4.2004).

Augenscheinlich ist zunächst die geringe Kongruenz der Listen in Abb. 2 und Abb. 4. Nur den Beatles gelang es, sich in beiden Listen auf den oberen Rängen zu platzieren (und dies auch nur aufgrund Regelung, dass der Verkauf von Doppelalben in der RIAA-Liste als Verkauf von zwei Alben gewertet wird). Einige der von den Fans und Kritikern hoch geschätzten Alben haben sich (zumindest in den USA) sogar nur ausgesprochen schlecht verkaufen 
können (Pet Sounds: 1,6 Mio., Highway 61 Revisited: 1,5 Mio., Astral Weeks: 500.000, Velvet Underground \& Nico: 311.000), ${ }^{1}$ während viele Bestseller von den Teilnehmern der Listenabstimmung nicht gewürdigt werden.

Im Vergleich mit den Verkaufszahlen tritt auch besonders deutlich hervor, was neben den gesamten 1980er Jahren in dem Listen-Kanon ebenfalls stark unterrepräsentiert ist. Während sich nämlich im Verkaufskanon einige wenige Musikerinnen finden lassen, sind Frauen, abgesehen von Bandmitgliedern von Fleetwood Mac und Velvet Underground, kaum auffindbar: unter den ersten hundert Alben stammen nur fünf von weiblichen Interpreten, davon nur eines unter den ersten fünfzig (Horses von Patti Smith).

Auf den ersten Blick gering erscheint auch der Anteil afro-amerikanischer Musiker in den Top 100 der Kanon-Liste (16\%). Dieser Wert relativiert sich allerdings etwas, wenn man in Betracht zieht, dass der Anteil der afroamerikanischen Bevölkerung in den USA mit ca. 13 \% noch darunter liegt. Vor allem in den Top 30 sind Musiker afro-amerikanischer Herkunft dennoch unterrepräsentiert (nur zwei Platzierungen), obwohl Musiker wie Michael Jackson und Whitney Houston in den Verkaufscharts äußerst erfolgreich sind. Entsprechend selten sind die Musikstile Soul, Blues und HipHop im Listen-Kanon vertreten. Weiterhin bleiben die laut Verkaufscharts sehr populären Musikstile Hard Rock/Heavy Metal und Country von den Listen errichtenden Fans und Kritikern unberücksichtigt - symptomatischer Weise zwei Genres, die eine größere Distanz zum Kunstdiskurs aufweisen (vgl. Bryson 1996). Daneben fehlen in der Kanonliste Soundtracks und Best-ofAlben, ganz offensichtlich, weil sie anders als die dort vertretenen Alben nicht als autonome »Werke « betrachtet werden.

Diese Befunde verlangen eine soziologische Erklärung (s. dazu ausführlich Appen/Doehring 2006: 24ff.): Ihr zufolge stellt sich dieser Albenkanon als Ergebnis des Handelns höher gebildeter weißer Männer des zweiten Lebensdrittels dar, die sich aufgrund ihrer Disposition und ihres Geschmacks zu einem Milieu um gewisse Zeitschriften oder Radiosender gruppiert haben. Ihr auf andere bezogenes Handeln gilt der quantitativ und qualitativ begründbaren Distinktion gegenüber kulturindustriell fabrizierter Massenware einerseits und der Unterscheidung von Musikgenres geringeren sozialen Prestiges andererseits. Gruppen wie die Beatles, Nirvana oder die Beach Boys entsprechen keiner der sich aus der Disposition der Teilnehmenden ergebenden Ausschlusskriterien; ihr Repertoire wird zwar mit kulturindustriellen Mechanismen gepflegt, doch haben sie »wenig genug « Alben verkauft, um sich von den Verkaufscharts absetzen zu können. Was aus der soziologischen

1 Quelle der Verkaufszahlen: http://www.rollingstone.com/news/story/5938174/ the_rs_500_greatest_albums_of_all_time, letzter Zugriff: 15.2.2008. 
Perspektive jedoch nicht begründet werden kann, ist die Bevorzugung bestimmter Alben: Warum favorisiert die Gruppe der Kanonisierer Revolver, nicht aber z.B. Let It Be? Hier kommt man an rezeptionsästhetischen Argumenten nicht vorbei. Kriterien, die zur Erklärung des hohen Wertes der aufgezählten »Klassiker « immer wieder herangezogen werden, sind u.a. Originalität, Innovation, persönlicher emotionaler Ausdruck, Vielfalt, Homogenität, Komplexität und Einfachheit, Authentizität und Qualitäten des Songwritings (ebd.: 29ff.). Gerade dies sind die zentralen ästhetischen Kriterien der Rockmusik der Jahre 1965-1969 (die wiederum nicht gering vom romantischen Kunstverständnis des 19. Jahrhunderts beeinflusst sind), welche den Kanon der Alben dominiert - der wiederum von einer soziologisch klassifizierbaren Gruppe erstellt wurde. Die enge Verflechtung von Soziologie und Ästhetik trägt somit erheblich zum Erkenntnisgewinn bei der Erforschung musikalischer Kanons bei.

\section{Zunahme der Kanonisierung}

Anhand der hier vorgestellten Kanonisierungsform der Albenlisten ist erkenntlich, wie weit sich populäre Musik von dem ihr unterstellten Wesen der gegenwartsbewussten, zweck- und gewinnorientierten Produktion von Vergnügsamkeit entfernt hat. Doch nicht nur auf diesem Feld wächst die popmusikalische Betätigung im historisierenden Modus. Generell scheint, so die vorgestellte These, ein Anwachsen des kanonisierenden Blicks auf populäre Musik in den letzten Jahren zuzunehmen; die im Folgenden kurz dargestellten Belege für eine derartige Annahme streuen über alle Medienformen und -gattungen hinweg.

\section{Audioformate}

Mittlerweile lassen sich zu (fast) jedem der in der Metaliste genannten Alben opulent gestaltete Neuausgaben finden. Diese sind in aller Regel mit Aufsätzen oder Interviews von Beteiligten, Zeitgenossen und/oder anderen so genannten Experten versehen, in denen anekdotisch die genialistischen Umstände der Entstehung, die zeitgenössische (sich selbstverständlich in der Rückschau überschwänglich gebende) Rezeption sowie die außerordentliche musikgeschichtliche Bedeutung des Albums herausgestellt werden. Derartig Bedeutung mimende Wiederveröffentlichungen, meist hochpreisig auf dem Markt platziert, werden zudem in Musikmagazinen, Wochen- und Tageszeitungen in Print- wie Onlineformaten, Radiosendungen und Fernsehbeiträ- 
gen wohlwollend begleitet. Dies generiert eine enorme öffentliche Aufmerksamkeit, die viele mit historischer Bedeutsamkeit der Alben gleichsetzen.

\section{Musikmagazine}

Als ein die mediale Resonanz verstärkender Effekt tritt hinzu, dass seit den 1990er Jahren eine Neugründungswelle von Online-Redaktionen sowie eine starke Anhäufung im Segment der Zeitschriften (und hier besonders bei Special-Interest-Zeitschriften, wozu Musikmagazine zählen) zu verzeichnen sind (vgl. Weischenberger/Malik/Scholl 2006: 34ff.). Somit vergrößert sich die Anzahl der Multiplikatoren der (zunächst) bloßen Nachricht über die Wiederveröffentlichung eines Albums, die im Kampf um die Lesergunst dann als Medien-Event inszeniert wird. Zusätzlich weitet sich die Zuständigkeit für (im weitesten Sinne) popmusikalische Themen über ehemalige Ressortgrenzen aus; neben Feuilleton und Musikmagazin »berichten« nunmehr Lifestyle-Magazine oder der ausufernde People-Journalismus über Menschen und Themen der Popmusik.

Bei den Musikmagazinen gibt es vielerlei Hinweise auf eine Zunahme historistischer Perspektiven. Zeitschriften mit Titeln wie Record Collector oder Good Times. Music from the $60 \mathrm{~s}$ to the $80 \mathrm{~s}$ stellen ihr verstärktes Interesse an einem geschichtsbewussten Umgang mit Popmusik noch betont unverstellt aus. Aber auch in dem Titel nach unverdächtigen Magazinen wird Popmusikgeschichte als Heldengeschichte anlassgebunden kanonisiert. So wirbt die deutsche Ausgabe des Rolling Stone im Jahr 2007 mit dem Slogan »Wir schreiben Musikgeschichte (etwa in der SZ vom 29.10.2007 auf S. 14) für eine Ausgabe (RS Nr. 11/2007), auf deren Titelblatt sich, immerhin 37 Jahre nach ihrer Trennung, die Beatles befinden. Und dies, weil ihr 42 Jahre alter Film Help auf DVD veröffentlicht wird - denn, so fragt Autor Joachim Hentschel (2007: 58), »haben die Beatles nicht nur die Musik, sondern auch das Kino verändert? « Ähnlich widmet der Metal Hammer das Cover sowie zahlreiche Seiten der Ausgabe 8/2007 dem zwanzigjährigen Jubiläum des Guns N'Roses-Albums Appetite For Destruction.

Dass im Rolling Stone Vergangenes nicht vergessen werden darf, beweisen auch die in loser Folge vorgestellten "Alten Meister « in gleichnamiger mehrseitiger Artikelserie sowie die mittlerweile auf sechs Seiten ausgedehn-

22007 waren auf dem Cover des deutschen Rolling Stone vertreten: vier "neue « (alle mit mindestens zwei veröffentlichten Alben) Bands bzw. Musiker (Arcade Fire, Amy Winehouse, M.I.A. und Devendra Banhart), zwei Kinostars (Borat und Johnny Depp) sowie die Altstars Paul Weller/Noel Gallagher, Jim Morrison, Travis (sicht- und hörbar ergrauend), Keith Richards, The Police, The Beatles und Bruce Springsteen. 
ten »Besprechungen « - Hymnen wäre angesichts des Tonfalls wohl die angemessenere Wortwahl - von Wiederveröffentlichungen älterer Alben. Die »besten « Alben wurden in den letzen zehn Jahren mehrfach bestimmt, mal in einer selbsterstellten 500er-Liste hierarchisiert (vgl. Rolling Stone 11/ 2004) oder auch in Listen nach einzelnen Jahrzehnten geordnet - und natürlich auf verschiedene Ausgaben verteilt - zusammengefasst (vgl. Rolling Stone 8-12/1997). Zusätzlich wird der erklärte Wert dieser Alben etwa durch das Begehen der Jahrestage ihrer Veröffentlichung und runder Geburts- oder Todestage der daran beteiligten Musiker genauso bestätigt wie in Artikeln über die Aufnahmeprozesse. Nicht minder wichtig sind intratextuelle Referenzen, die den »großen Alben« von Musikern in Interviews oder von den Redakteuren in eigenen Artikeln und Rezensionen immer wieder erwiesen werden.

\section{Buchmarkt}

Auch auf dem Buchmarkt zeigt die ansteigende Zahl der Neuveröffentlichungen zum Thema, dass Popmusikgeschichte als Kanon existiert - bestens repräsentiert in der etablierten Form des empfehlenden Konzertführers (vgl. etwa Dimery 2006; Freeman 2007; Levy 2005; Schütte 2004). Des Weiteren finden sich zu einzelnen Alben populärwissenschaftliche Reihen (vgl. die derzeit 53-bändige Serie $331 / 3)^{3}$ oder wissenschaftlichen Monografien. Ebenso werden die Musiker oder Bands in neuen oder neu aufgelegten Biographien geehrt, bspw. im Reclam Verlag die Bücher über Bob Dylan (Detering 2007), die Rolling Stones (Diez 2007) und die Beatles (Kemper 2007). Eine neue Sparte eröffnen diejenigen Publikationen, welche die kanonisierten Alben in reißerischem Tonfall dekanonisieren wollen (DeRogatis/Carillo 2006; Nonhoff 2005) oder aber dem existierenden Kanon einen alternativen Blick auf die Popgeschichte entgegenstellen (Büsser 2004).

Die Süddeutsche Zeitung beispielsweise erkannte diese offensichtliche Absatzmöglichkeit und veröffentlichte nach ihrer erfolgreichen Buchreihe SZ Bibliothek die SZ Diskothek, eine nach einzelnen Jahren geordnete Edition mit CD und begleitenden Texten, in der »die großen Momente der Popmusik, in Reihe gebracht, heute eine Chronik unserer Zeit ergeben, die unmittelbarer und wahrhaftiger ist als jene, die bei der Betrachtung politischer

3 Der Continuum-Verlag, in dem die Reihe seit 2003 erscheint, wirbt auf seiner Website: »It was only a matter of time before a clever publisher realized that there is an audience for whom Exile on Main Street or Electric Ladyland are as significant and worthy of study as The Catcher in the Rye or Middlemarch « (Continuum 2008, zitiert wird die New York Times Book Review). Für die Jahre 2008 und 2009 sind weitere 30 Bände angekündigt. 
Ereignisse entsteht «(Oehmke/Waechter 2005: 17). Allerdings werden ebendiese Momente - woanders etablierten Künstlern wie Bob Dylan oder den Beatles zugestanden - ausgespart zugunsten »sperriger Künstler «, denn hier gelte es, Popmusik wiederzuentdecken, »auf ein Neues, frei von Ideologie und Vorurteilen « (ebd.). Indes: das Fehlen dieser arrivierten Musiker erklärt man zwar mit der intimen Vertrautheit ihres Schaffens (»deren Hits können wir immer noch im Schlaf singen«) - rechtliche und ökonomische Aspekte der Verwertung, die in erster Linie verantwortlich für diese Lücke sein dürften, finden keine Erwähnung.

Andere Medienformen spiegeln ebenfalls den Boom des geschichtlichen Blicks auf Popmusik. Auf DVD werden Konzerte und Dokumentationen ebenjener Musiker veröffentlicht, die auch in den Albenlisten weit oben stehen. Ein Beispiel: für die Suche nach »Bob Dylan« gibt Amazon.de für die Jahre 2005-2007 die Zahl von 27 DVDs an (ausgelassen wurden in dieser Zählung Spielfilme und Lehrvideos); der Peaklevel liegt im Jahr 2006, in welchem Dylan seinen 65. Geburtstag feierte. Fernsehsender wie ARTE präsentieren Dokumentationen über einzelne Alben der Besten-Listen oder arbeiten sich thematisch am vierzigsten Jubiläum des »Sommers der Liebe« ab. Für das Internet lassen sich zusammenfassend zwei wichtige Funktionen erkennen: zum einen die des bündelnden Spiegels der in den anderen Medien stattfindenden Diskurse über Popmusikgeschichte, zum anderen als interaktives Forum, auf dem die Nutzer diese Diskurse debattieren. Von großem Wert hinsichtlich der Erforschung existierender Kanons und ihrer Begründungen erscheinen etwa die in jedem Forum erörterten Listen der »besten« Alben aller Zeiten resp. einzelner Dekaden oder Jahre.

Die Frage nach dem Grund dieser ansteigenden Menge an Kanondebatten soll im Folgenden in vier Thesen beantwortet werden: (1) Medienwechsel zwingen zum Überdenken der Bestände, (2) es gibt immer mehr Teilnehmer am zunehmend gesellschaftlich akzeptierten Popmusikdiskurs, (3) Menschen brauchen Orientierung in neuen und unüberschaubaren Lagen und (4) die Industrie weiß dies zu nutzen.

1. Jeder Besitzer eines neuen Festplattenspielers kennt das Dilemma, welche seiner CDs er zuerst auf den Player einlesen soll und welche auf gar keinen Fall fehlen dürfen. Derzeit findet ein Medienwechsel vom CD- zum mp3-Format statt, der die musikalischen Bestände einer Revision unterwirft. Der letzte Wechsel im Speicherungsformat von Musik war der Wechsel vom LP- zum CD-Format Mitte der 1980er Jahre. Damals war zu beobachten, dass Aufnahmen bereits etablierter Musiker und Bands zuerst in das neue Format überführt wurden, andere hingegen auf eine 
Wiederveröffentlichung auf CD länger warten mussten. Dies hatte Folgen für die Käufer: Neben den damaligen aktuellen Charts-Interpreten konnte man nun die Beatles wieder bzw. für einige Hörer zum ersten Mal auf einem neuen Medium kaufen. Zugrunde liegt dem ein Mentalitätswandel des bisher auf Aktualität gepolten Käufers, der nun ermöglichte, das Alte als wertvoll(er) neben die Neuerscheinungen in das Regal zu stellen.

Dieses Bewusstsein erlaubt auch heute, etwa das iPhone mit »alter « Musik zu füllen. Dennoch ist es interessant, die Entwicklung dieses Medienwechsels zum mp3-Format zu verfolgen. Zwar ist bald, vielleicht bereits heute, jede irgendwann einmal aufgezeichnete Musik in digitaler Form erhältlich, die Frage wird sich jedoch alleine schon aus technischen und personalen Kapazitätsproblemen stellen, welche Musik Einzelne oder bestimmte Gruppen als wertvoll genug für eine Aufbewahrung erachten. Und die Erfahrung lehrt, dass hier existierende Kanons samt ihrer Hüter mit der ihnen eigenen Resistenz gegenüber schnellem Wandel als wichtiges Lenkungsorgan in diesen Prozess eingreifen werden.

2. Wenn sie denn überhaupt je eine Musik der Jugend war, so ist Popmusik heute mehr denn je eine Musik der Jüngeren und Älteren. Das Altern der ersten mit Pop sozialisierten Generation der um 1950 Geborenen sowie die Ausweitung der Adoleszenz bis in das als erwachsen geltende Lebensalter hinein führen zu einer gesellschaftlich als legitim(er) betrachteten Beschäftigung mit Pop. Ein Beispiel: Im Feuilleton der ZEIT, wo die »100 Klassiker der Modernen Musik « wöchentlich vorgestellt werden, findet sich neben Bergs Wozzeck oder Bartóks 4. Streichquartett mittlerweile natürlich auch Revolver der Beatles (und ohne großes Aufsehen Slayers Reign In Blood). War die Debatte über die Inhalte des Plattenkanons beispielsweise in den Siebziger Jahren einer relativ kleinen Gruppe von Spezialisten vorbehalten, so nimmt heute, davon ist auszugehen, nicht nur die Zahl der Debattierenden zu, sondern erst recht die Menge derjenigen, die mit diesen Listen in den Medien konfrontiert werden. Die Zahl der (vorerst: indirekten) Kanonteilnehmer ist also gestiegen: die »Alten« verbleiben im Diskurs, die Jüngeren und Spätadoleszenten können ihm kaum entgehen. Ein weiterer, für die Musikindustrie wichtiger Aspekt dieser Zielgruppenvergrößerung ist die mit dem höheren Alter korrelierende Menge an Geld, welches für musikbezogene Ausgaben zur Verfügung steht (s.u.).

3. Gerhard Schulze (1992: 62) wies darauf hin, dass die Mitglieder moderner Gesellschaften aufgrund des vorherrschenden Modus der Unsicherheit von einem »ästhetischen Anlehnungsbedürfnis« getrieben werden, sich in 
Gruppen mit typischen Handlungsweisen zusammenzuschließen. Je gröBer das sie umgebende Chaos sei, desto eher fühle man sich zur Inanspruchnahme kollektiver Orientierungshilfen getrieben. Angesichts der unüberblickbar großen Menge an Musik und - bedingt durch den Medienwechsel auf mp3 - ihrer allgegenwärtigen, wenn auch mitunter illegalen Verfügbarkeit ist davon auszugehen, dass der Einzelne froh ist, auf die Frage nach dem besten Album / der besten Band / dem besten Sänger usw. im Kanon ein »ästhetisches Geleit« zur Verfügung zu haben. Denn kanonische Sammlungen bürgen für einen »Kulturschatz«, der von Experten meines Vertrauens (hier den Kritikern und Lesern des Musikmagazins, der Zeitung, der Website, dem social network meiner Wahl) zusammengestellt wurde.

Hinzu kommt, dass nicht nur das »Chaos « größer zu werden scheint, sondern sich die Anteile von Arbeit und Freizeit immer weiter verschieben. Zwar wird auf Grund technischer Entwicklung Ort und Zeit der Arbeit flexibilisiert (bspw. kann die Büropost unterwegs vom Mobiltelefon oder -computer aus erledigt werden), dringt dadurch aber in Bereiche, die ehemals den Arbeitnehmern zur freien Verfügung gestanden hatten (etwa wenn der letzte Abruf der Emails vor dem Zubettgehen erfolgt). Gerade für die musikaffinen Kulturschaffenden des Creative-Class-Concepts (vgl. überblickend Barber-Kersovan 2007) oder der selbsternannten »Digitalen Bohème « (Friebe/Lobo 2006) kann dieser Kanon der Alben - im neoliberalen Zeitgeistsprech - »kosteneffizient « und »zielgerichtet « dem vorgeblichen Bildungserwerb der »besten« Musik der zurückliegenden Jahre dienen. Und, quasi als »Gewinnmitnahme on top«, können nun mit derart kompetent gefüllten Festplatten Freunde und Kollegen beeindruckt werden.

Eine Bemerkung am Rande: Es bleibt abzuwarten, ob eine lebensnahe Bedeutsamkeit dieser Musik durch Vollständigkeit der Daten allein substituiert werden kann. Der Vergleich mit einem anderen Kulturfeld, der mit Gesamtausgaben bestückten Schrankwand bürgerlicher Wohnstuben, legt nahe, dass es sich hier um nicht mehr als eine moderne Form des Kulturgrabs handelt. Denn Hand aufs Herz: wann hat man das letzte Mal Sgt. Pepper's gehört, wann Faust gelesen?

4. Die »Welt in einer Liste« bietet für die Musikindustrie den geschätzten Vorteil, diese als wertvoll erkannten Kulturdenkmäler rational herstellen, bewerben und verkaufen zu können. Die Zielgruppe der eher älteren, finanziell besser gestellten Männer sowie ihre Vorlieben für optisch wie haptisch ansprechende und Werthaltigkeit signalisierende Verpackungen 
der Wiederveröffentlichungen, gerne auch als teuere Super-Audio-CDEditionen, versinnbildlicht die Neuausgabe von Pet Sounds der Beach Boys idealtypisch. Das Album ist in fünf verschiedenen Ausführungen in der Preisspanne von 16 bis 50 Euro erhältlich, wahlweise kann man eine Einzel-CD mit Bonustracks, eine Doppel-CD mit Mono- und Stereo-Mix, eine CD mit samtenem Cover inkl. DVD und Dolby Surround-Mix (in 96 $\mathrm{kHz}$ ) oder das vier CD-Längen umfassende Pet-Sounds-Sessions Box-Set erwerben.

Die vordergründigen Anlässe solcher Wiederveröffentlichungen sind in der Regel Jubiläen (im Falle von Pet Sounds war es das vierzigjährige Jubiläum der Erstausgabe). Diese werden wiederum Auslöser der bereits oben beschriebenen Berichterstattung in den Medien, die für die Verfestigung des Kanons so wichtig ist. Denn derart wird nun Altes unter dem Signum der Aktualität, welches systemtheoretisch argumentiert die massenmediale Leitdifferenz des binären Codes Information/Nicht-Information unter dem Faktor Zeit ist, präsentiert und kann somit kulturelle Geltung beanspruchen.

Auch für den Tonträgermarkt, der sich in einem dramatischen Schrumpfungsprozess befindet, bietet das »Meisterwerk « aus Sicht der Musikindustrie drei enorme Vorteile. Es hat erstens keine Produktionskosten mehr einzuspielen; die Studiokosten und Vorauszahlungen an die Musiker entfallen, die Promotionkosten sind aufgrund des Bekanntheitsgrades geringer als bei Debütanten. Zweitens ist es einfacher, bereits bekannte Musik zu verkaufen als unbekannte. Die Scheu vor dem Neuklang auf Rezipientenseite resultiert sicherlich nicht zuletzt aus der wie gleichgeschaltet klingenden Bespielung durch die Formatradios; ein weiterer Grund dürfte im höheren Alter und dem somit verfestigten Musikgeschmack der Kanonteilnehmer liegen. Und schließlich bedeutet die Zuschreibung des Werk-Charakters an diese Alben eine wichtige Waffe im Kampf gegen den Download - denn »Kunstwerke« lassen sich nun einmal schlecht, noch dazu in vergleichsweise schlechter Klangqualität und ohne zugehöriges Artwork, aus dem Netz ziehen. Der letzte Punkt weist auf eine grundlegende Voraussetzung für das Funktionieren dieses Marktes hin. Nämlich erst dieses geschichts- wie kunst- und somit vorgeblich qualitätsorientierte Musikverständnis ermöglicht es der Musikindustrie, dreißig und mehr Jahre alte Musik an den (in der Regel dreißig und mehr Jahre alten) Mann zu bringen. 


\section{Diskussion}

Während die Neue Musik zwar einen sicheren Platz im akademischen Kanon, dabei aber wenig Bedeutung für den pädagogischen und den RepertoireKanon hat, ist die Kanonbildung in der Pop- und Rockmusik heute stark vorangeschritten und verfestigt. Diese Musik wurde aus der Geschichtslosigkeit in einen Zeiten überdauernden und das Musikleben nachhaltig beeinflussenden Diskurs der Kenner, Experten und sonstigen Meinungsmacher überführt. Sie hat den Sprung von lebensnahen Funktionen (vgl. Polaschegg 2005: 124f.), von der situationsgebundenen Produktion und Reproduktion zum autonom rezipierten Musikstück vollzogen. Der entstandene Kanon gibt Impulse für die Meinungsvermittler und -multiplikatoren in Schule, Medien und Kulturpolitik. Sein Erfolg begründet sich durch die Vorteile der orientierenden Funktion (sowohl in Bezug auf Identität wie auch auf Distinktion), der damit verbundenen Zeitersparnis beim Kulturerwerb sowie des ökonomischen Nutzens für Produzenten und Vermittler.

Negativ behaftet ist und bleibt ein Kanon aufgrund der starken Einengung und Standardisierung des musikalisch Möglichen und der Durchsetzung der Maßstäbe einer zwar kleinen, aber kulturell mächtigen Gruppe für viele - zumal die auf diese Weise durchgesetzten Werte viel mehr betreffen als nur die Musik, darunter Geschlechterrollen und ganze Weltsichten. So fehlt mit Alben aus den 1980er Jahren bspw. die gesamte postmoderne Sicht auf Musik zugunsten eines Authentizitäts-Anspruchs, der typisch für die 1960er Jahre ist. Daneben ist zu bedenken, dass das Geld, welches die Musikindustrie und die Konsumenten in die Bewerbung und den Kauf von mittlerweile als Klassiker geltenden Alben und Musikern investieren, für die Förderung einer lebendigen, innovativen Gegenwartskultur nicht mehr zur Verfügung steht.

Folgt man Walter Wioras (1969b: 301f.) Beschreibung des Historismus als Dominanz historischer Aspekte vor allen anderen, so müsste man zusammenfassend zu dem Ergebnis gelangen, die gesellschaftliche Beschäftigung mit Pop finde dieser Tage unter »Vorherrschaft historischer Verhaltens- und Betrachtungsweisen « statt. Die Welt der Popmusik wäre also ordentlich kanonisiert und geschichtlich in Reih und Glied gestellt: »no time for losers«.

Dagegen spricht jedoch, dass ein Kanon, wie er hier für das Albumformat in der Pop- und Rockmusik nachgewiesen wurde, in der ausdifferenzierten Moderne wohl kaum eine normative Kraft, gleichsam eines kollektiven Gedächtnisses aller, entwickeln kann. Nur sektional auf Subsysteme beschränkt erlangt er dort Gültigkeit, wo er sich in den praktischen Lebens- 
vollzug sinnvoll eingliedern lässt (vgl. Hahn 1998; Heydebrand 1998). So ist etwa dieser Kanon für die Bravo völlig irrelevant, Lifestyle-Zeitschriften mit eigenem Musikteil nehmen inn kaum zur Kenntnis und selbst in Musikzeitschriften wie Intro oder Spex werden (falls überhaupt) andere Alben kanonisiert. Dafür verantwortlich sind vor allem demographische Unterschiede in Alter, Geschlecht und Bildung, aber auch der ideologische Habitus und damit zusammenhängend das Musikverständnis. Nur dort, wo ein historisch orientiertes Musikverständnis den praktischen Musikvollzug begleitet, ist Kanonisierung möglich. Ein eher ahistorisch, d.h. gegenwartsbezogenes und auf die subjektive Valenz gerichtetes Musikverständnis, wie es bei jüngeren Hörern oder »Gelegenheitskäufern« (IFPI 2008: 39) häufig vorzufinden ist, führt dagegen zu einer Ignoranz oder Indifferenz gegenüber den kanonisierten »Meisterwerken«.

Dennoch ist vor einer Unterschätzung dieses Kanons zu warnen. Obgleich es nun scheinen mag, als handle es sich bei Kanonteilnehmern nur um eine kleine Gruppe neben vielen anderen, die diesen Kanon eben nicht aktiv gestalten oder wahrnehmen, muss man auf seine Sedimentation in das öffentliche Bewusstsein hinweisen. Auf der von vielen Zeitgenossen als »Wissensquelle « geschätzten Website Wikipedia etwa kann man zu allen der in der Liste befindlichen Alben mehr oder minder gute Artikel finden - und in keinem davon fehlt der Hinweis auf die Platzierung des jeweiligen Albums in einer der von den relevanten Musikmagazinen herausgegebenen Listen. Wiewohl davor gewarnt wurde und wird: die Macht der Zahl als »Kanon der Aufklärung « (Horkeimer/Adorno 1988: 13) besteht weiterhin, die Gleichung hohe Platzierung = hohe Qualität scheint noch immer aufzugehen.

Mit diesem kritischen Bewusstsein für die Vor- und Nachteile kann und sollte der Kanon durchaus auch Gegenstand der Musikvermittlung in den Schulen sein. Es ist nur zu begrüßen, wenn die Schüler anhand solcher gemeinhin als Meilensteine geltenden Aufnahmen die Tradition kennenlernen, vor deren Hintergrund gegenwärtige populäre Musik spielt. Der Vorteil der Orientierungsfunktion eines Kanons sollte also genutzt werden. Unsere Überlegungen haben aber gezeigt, wie wichtig es ist zu erkennen, was im Kanon unterrepräsentiert ist und an welchen medialen und gesellschaftlichen Mechanismen dies liegt. Zwingend erforderlich ist also die gleichzeitige Aufklärung über jene Mechanismen, über die sozialen und machtpolitischen Funktionen des Kanons sowie die das Musikverständnis betreffenden Grundlagen solcher Kanonisierung. Auf diese Weise könnten Schülerinnen und Schüler ihre eigenen Werte und Ausschlüsse reflektieren, was im Idealfall zu größerer Offenheit gegenüber den Alternativen, zu Pluralität und 
Toleranz führt. Kanonbildung ist nicht zu vermeiden - umso wichtiger ist ein aufgeklärter und kritischer Umgang mit ihr.

\section{Literatur}

Adorno, Theodor W. (1968). Einleitung in die Musiksoziologie. Zwölf theoretische Vorlesungen. Reinbek: Rowohlt.

Appen, Ralf von / Doehring, André (2000). »Kanonisierung in der Pop-/Rockmusik oder: Warum Sgt. Pepper? Zur ästhetischen Beurteilung von Pop-/Rock-LPs in 100er Listen. « In: Populäre Musik im kulturwissenschaftlichen Diskurs. Hg. v. Helmut Rösing und Thomas Phleps (= Beiträge zur Popularmusikforschung 25/26). Karben: Coda, S. 229-249.

Appen, Ralf von / Doehring, André (2006). »Nevermind The Beatles, Here's Exile 61 and Nico: 'The Top 100 Records of All Time - A Canon of Pop and Rock Albums from a Sociological and an Aesthetic Perspective. "In: Popular Music 25, Vol. 1, S. 21-39.

Barber-Kersovan, Alenka (2007). "Creative class, creative industries, creative city. Ein musikpolitisches Paradigma. «In: Sound and the City. Populäre Musik im urbanen Kontext. Hg. v. Dietrich Helms und Thomas Phleps (= Beiträge zur Popularmusikforschung Bd. 35). Bielefeld: Transcript, S. 11-30.

Belz, Carl (1969). The Story of Rock. New York: Oxford University Press.

Bourdieu, Pierre (1984). Die feinen Unterschiede. Kritik der gesellschaftlichen Urteilskraft. Frankfurt/M.: Suhrkamp (3. Aufl.).

Bryson, Bethany (1996). ")Anything but Heavy Metal.< Symbolic Exclusion and Musical Dislikes. «In: American Sociological Review 61, S. 884-899.

Büsser, Martin (2004). On the wild side. Die wahre Geschichte der Popmusik. Hamburg: Europäische Verlagsanstalt.

Cohn, Nik (1969). Pop from the Beginning. London: Weidenfeld and Nicolson.

Continuum (2008). "Series 33 1/3." Online unter: http://www.continuumbooks. com/Series/default.aspx?ImprintID=2\&CountryID=1 (Zugriff: 13.2.2008).

DeRogatis, Jim / Carrillo, Carmél (Hg.) (2006). Hall of Shame. Die größten Irrtümer in der Geschichte des Rock'n'Roll. Berlin: Rogner \& Bernhard.

Detering, Heinrich (2007). Bob Dylan. Stuttgart: Reclam.

Dietz, Georg (2007). The Rolling Stones. Stuttgart: Reclam.

Dimery, Robert (Hg.) (2006). 1001 Alben. Musik, die Sie hören sollten, bevor das Leben vorbei ist. Zürich: Edition Olms.

Dollase, Rainer / Rüsenberg, Michael / Stollenwerk, Hans J. (1986). Demoskopie im Konzertsaal. Mainz: Schott.

Ehrenzweig, Anton (1982). Ordnung im Chaos. Das Unbewußte in der Kunst. Köln: Kindler (engl. Original 1967).

Eisen, Jonathan (Hg.) (1969). The Age of Rock. Sounds of the American Cultural Revolution. New York: Vintage.

Freeman, Phil (Hg.) (2007). Marooned. The Next Generation of Desert Island Discs. Cambridge: Da Capo.

Friebe, Holm / Lobo, Sascha (2006). Wir nennen es Arbeit. Die digitale Bohème oder: Intelligentes Leben jenseits der Festanstellung. München: Heyne.

Hahn, Alois (1998). »Einführung. «In: Kanon Macht Kultur. Theoretische, historische und soziale Aspekte ästhetischer Kanonbildungen. Hg. v. Renate von Hey- 
debrand (= Germanistische Symposien Berichtsbände XIX). Stuttgart, Weimar: Metzler, S. 459-466.

Hentschel, Joachim (2007). "The Beatles on film. Wie John, Paul, George \& Ringo den Pop ins Popcorn setzten. « In: Rolling Stone, Nr. 11 (dt. Ausgabe), S. 58-67.

Heydebrand, Renate von (1998). "Kanon Macht Kultur - Versuch einer Zusammenfassung. « In: Kanon Macht Kultur. Theoretische, historische und soziale Aspekte ästhetischer Kanonbildungen. Hg. v. ders. (= Germanistische Symposien Berichtsbände XIX). Stuttgart, Weimar: Metzler, S. 612-625.

Horkheimer, Max / Adorno, Theodor W. (1988). Dialektik der Aufklärung. Philosophische Fragmente. Frankfurt/M.: Fischer.

IFPI (2008). »Jahreswirtschaftsbericht 2007.« Online unter: www.musikindustrie. de/uploads/media/7_musikkaeufer.pdf (Zugriff: 28.5.2008).

Kaden, Christian (1985). Musiksoziologie. Wilhelmshaven: Heinrichshofen.

Kemper, Peter (2007). The Beatles. Stuttgart: Reclam.

Konrad, Ulrich (2004). »Mozart. "In: Die Musik in Geschichte und Gegenwart. Personenteil, Bd. 6. Hg. v. Ludwig Finscher. Kassel, Stuttgart: Bärenreiter/Metzler (2. Aufl.), Sp. 591-758.

Levy, Joe (Hg.) (2005). Rolling Stone - The 500 Greatest Albums of All Time. New York: Wenner Books.

Moore, Allan F. (1997). The Beatles. Sgt. Pepper's Lonely Hearts Club Band (= Cambridge Music Handbooks). Cambridge: Cambridge University Press.

Nonhoff, Sky (Hg.) (2005). Don't believe the hype! Die meistüberschätzten Platten der Popgeschichte. Frankfurt/M.: Fischer.

Oehmke, Philipp / Waechter, Johannes (2005). "Der ewig coole Klang. Warum die Krise der Popmusik die Chance eröffnet, ihre großen Momente neu zu entdecken. «In: Süddeutsche Zeitung, Nr. 132, vom 11./12. Juni, S. 17.

Polaschegg, Nina (2005). Populäre Klassik - Klassik populär. Hörerstrukturen und Verbreitungsmedien im Wandel. Köln: Böhlau.

Regev, Motti (1994). »Producing Artistic Value. The Case of Rock and Popular Music.«In: The Sociological Quarterly 35, Vol. 1, S. 85-102.

Rösing, Helmut (2002). »Populäre Musik und kulturelle Identität. Acht Thesen. «In: Heimatlose Klänge? Regionale Musiklandschaften - heute -. Hg. v. Thomas Phleps (= Beiträge zur Popularmusikforschung 29/30). Karben: Coda, S. 11-34.

Salganik, Matthew J. / Dodds, Peter Sheridan / Watts, Duncan J. (2006). »Experimental Study of Inequality and Unpredictability in an Artificial Cultural Market." In: Science, Vol. 311 (10 February), S. 854-856.

Schulze, Gerhard (1992). Die Erlebnisgesellschaft. Kultursoziologie der Gegenwart. Frankfurt/M., New York: Campus.

Schütte, Uwe (2004). Basisdiskothek Rock und Pop. Stuttgart: Reclam.

Smith, Chris (2007). 100 Albums that Changed Popular Music. A Reference Guide. Westport: Greenwood.

Smudits, Alfred (2002). Kunst und Kommunikationstechnologien im Wandel (= Musik und Gesellschaft 27). Wien: Braunmüller.

Turner, Victor (2005). Das Ritual: Struktur und Antistruktur. Frankfurt/M.: Campus (2. Aufl.; engl. Original 1969).

Ullmaier, Johannes (1995). Pop shoot Pop. Über Historisierung und Kanonbildung in der Popmusik. Rüsselsheim: Frank Hofmann.

Wiora, Walter (Hg.) (1969a). Die Ausbreitung des Historismus über die Musik (= Studien zur Musikgeschichte des 19. Jahrhunderts 14). Regensburg: Bosse. 
Wiora, Walter (1969b). »Grenzen und Stadien des Historismus in der Musik.«In: Die Ausbreitung des Historismus über die Musik. Hg. v. dems. (= Studien zur Musikgeschichte des 19. Jahrhunderts 14). Regensburg: Bosse, S. 299-327.

\begin{abstract}
Instead of being considered ahistorical and »just for the moment, « pop and rock productions are increasingly regarded as autonomous works of cultural significance just as "serious" or "classical « music is. After some introductory reflections concerning the terminology, we address sociological, psychological and economic functions and mechanisms of canonisation. We point out similarities between the popular and the classical realm and identify an underlying common sensibility that can be seen as the base of canonisation. Subsequently, we describe and analyse the pop and rock albums which music critics have succeeded in establishing as the current canon.

We prove empirically that canonising views on popular music have increased over the last years, both in terms of supply and demand. Finally, we discuss differences between the »high brow« and the popular canon as well as pedagogic implications of canonisation. We interpret the current situation according to aspects of historicism and ahistoricity and conclude by offering a prognosis for future development.
\end{abstract}

